\title{
ON THE LOOMIS-SIKORSKI THEOREM FOR MV-ALGEBRAS WITH INTERNAL STATE
}

\author{
A. DI NOLA, A. DVUREČENSKIJ ${ }^{\bowtie}$ and A. LETTIERI
}

(Received 20 April 2010; accepted 27 December 2010)

Communicated by M. G. Jackson

\begin{abstract}
In Flaminio and Montagna ['An algebraic approach to states on MV-algebras', in: Fuzzy Logic 2, Proc. 5th EUSFLAT Conference, Ostrava, 11-14 September 2007 (ed. V. Novák) (Universitas Ostraviensis, Ostrava, 2007), Vol. II, pp. 201-206; 'MV-algebras with internal states and probabilistic fuzzy logic', Internat. J. Approx. Reason. 50 (2009), 138-152], the authors introduced MValgebras with an internal state, called state MV-algebras. (The letters MV stand for multivalued.) In Di Nola and Dvurečenskij ['State-morphism MV-algebras', Ann. Pure Appl. Logic 161 (2009), 161-173], a stronger version of state MV-algebras, called state-morphism MV-algebras, was defined. In this paper, we present the Loomis-Sikorski theorem for $\sigma$-complete MValgebras with a $\sigma$-complete state-morphism-operator, showing that every such MV-algebra is a $\sigma$-homomorphic image of a tribe of functions with an internal state induced by a function where all the MV-operations are defined by points.
\end{abstract}

2010 Mathematics subject classification: primary 06D35; secondary 03B50, 03 G12.

Keywords and phrases: MV-algebra, Boolean algebra, state MV-algebra, internal state, state-morphism MV-algebra, state-morphism-operator, Loomis-Sikorski theorem, tribe.

\section{Introduction}

MV-algebras were introduced in the late fifties by Chang [3] as algebraic semantics for Łukasiewicz many-valued logic; the letters MV stand for multi-valued. Nowadays $\mathrm{MV}$-algebras enter in many areas of mathematics and its applications, including quantum structures; see, for example, [12]. The seminal paper that is crucial for the theory of MV-algebras is that of Mundici [23], concerning the categorical equivalence of the variety of MV-algebras and the category of unital $\ell$-groups; for an overview of MV-algebras see [4].

This research was supported by the Center of Excellence SAS-Quantum Technologies, ERDF OP R \& D Projects CE QUTE ITMS 26240120009 and meta-QUTE ITMS 26240120022, the grant VEGA No. 2/0032/09 SAV, by the Slovak Research and Development Agency under the contract APVV-0071-06, Bratislava, and by the Slovak-Italian project SK-IT 0016-08.

(C) 2011 Australian Mathematical Publishing Association Inc. 1446-7887/2011 \$16.00 
The Loomis-Sikorski theorem was proved independently by Loomis [22] and Sikorski [29]; see, for example, [30]. It states that each $\sigma$-complete Boolean algebra is a $\sigma$-epimorphic image of a $\sigma$-algebra of subsets of some set $\Omega$. This result was extended to $\sigma$-complete $\mathrm{MV}$-algebras in [9, 25]; see also [1]. In this case, every $\sigma$-complete $\mathrm{MV}$-algebra is a $\sigma$-epimorphic image of a tribe of $[0,1]$-valued functions on a set $\Omega$, where the $\mathrm{MV}$-algebraic operations among functions are defined by points. This result was also extended to monotone $\sigma$-complete effect algebras in [2].

Forty years after the appearance of MV-algebras, Mundici [24] presented an analogue of probability measure for MV-algebras, called a state, as an averaging process for formulas in Łukasiewicz logic. In the last decade, the theory of states on MV-algebras and relative structures has been intensively studied; see, for example, $[13,16,19,20,26-28]$. We emphasize that a state is a proper notion for quantum structures; see [12].

Recently, Flaminio and Montagna in [14, 15] extended the language of MValgebras, adding a unary operation $\tau$, called an internal state or a state-operator. Such $\mathrm{MV}$-algebras are called state MV-algebras. We recall that modality $\operatorname{Pr}$ (interpreted as probably) in many-valued logic has the following semantic interpretation: the probability of an event $a$ is the truth value of $\operatorname{Pr}(a)$. Furthermore, if $s$ is a state, then $s(a)$ is interpreted as an average of the appearances of the many-valued event $a$.

State MV-algebras have been intensively studied; see, for example, [5-7]. There is a special type of state-operators: state-morphism-operators, which are state-operators that are also MV-homomorphisms. In [5], we characterized the set of subdirectly irreducible state-morphism MV-algebras (we note that there is still no characterization of subdirectly irreducible state MV; see [14]). In [7, 8], we described different varieties of state MV-algebras; in particular, we showed that if $A$ is an MV-algebra, $\tau$ is a stateoperator and $\tau(A) \in \mathrm{V}\left(S_{1}, \ldots, S_{n}\right)$, then $\tau$ is a state-morphism-operator; we recall that $\mathrm{V}\left(S_{1}, \ldots, S_{n}\right)$ is the variety of MV-algebras generated by $S_{1}, \ldots, S_{n}$ and $S_{i}$ is the MV-algebra of the form $S_{i}=\{0,1 / i, 2 / i, \ldots, i / i\}$.

In this paper, we show that every $\sigma$-complete state-morphism MV-algebra $(A, \tau)$ is an epimorphic image of an appropriate tribe of functions on some set $\Omega$ with a state-morphism-operator induced by a function from $\Omega$ into itself. This gives a new variant of the Loomis-Sikorski theorem for $\sigma$-complete state-morphism MV-algebras with internal state.

The paper is organized as follows. In Section 2, we give elements of the theory of MV-algebras. We mention general comparability, which every $\sigma$-complete MValgebra satisfies, and recall some basic representations of MV-algebras satisfying general comparability. Section 3 presents state MV-algebras and state-morphism $\mathrm{MV}$-algebras. We give some characterizations of semisimple state-morphism MValgebras and show that each state-morphism-operator is induced by some idempotent function $g$, which we may assume is continuous on some compact Hausdorff topological space. The main body of the article is Section 4, where the LoomisSikorski theorem and its variants, including a continuous variant, are proved. The last section gives some alternative proofs of Theorem 3.7 for special cases. 


\section{MV-algebras and general comparability-properties}

We recall that an $M V$-algebra is an algebra $\left(A ; \oplus,{ }^{*}, 0\right)$ of signature $\langle 2,1,0\rangle$, where $(A ; \oplus, 0)$ is a commutative monoid with neutral element 0 and the following conditions hold for all $x, y \in A$ :

- $\quad\left(x^{*}\right)^{*}=x$;

- $x \oplus 1=1$, where $1=0^{*}$;

- $\quad x \oplus\left(x \oplus y^{*}\right)^{*}=y \oplus\left(y \oplus x^{*}\right)^{*}$.

We define an additional total operation $\odot$ on $A$ via $x \odot y:=\left(x^{*} \oplus y^{*}\right)^{*}$.

Suppose that $(G, u)$ is an Abelian $\ell$-group with a strong unit $u \geq 0$, that is, $G$ is a lattice-ordered group and for all $g \in G$, there is a positive integer $n$ such that $g \leq n u$. Then a prototypical example of an MV-algebra is

$$
A=\left(\Gamma(G, u) ; \oplus,^{*}, 0\right)
$$

where $\Gamma(G, u):=[0, u], \Gamma$ being the Mundici functor, $g_{1} \oplus g_{2}:=\left(g_{1}+g_{2}\right) \wedge u$ and $g^{*}:=u-g$; indeed, by [23], every MV-algebra is isomorphic to some $\Gamma(G, u)$.

We recall that an ideal of an MV-algebra $A$ is a nonempty subset $I$ of $A$ such that if $a \leq b$ and $b \in I$, then $a \in I$, and also if $a, b \in I$, then $a \oplus b \in I$. An ideal $I$ is maximal if $I \neq A$, and also, if $J$ is an ideal and $I \subseteq J \neq A$, then $I=J$. The dual notion to an ideal is a filter. We define the radical of $A$ by $\operatorname{Rad}(A):=\bigcap\{I \in \mathcal{M}(A)\}$, where $\mathcal{M}(A)$ is the set of all maximal ideals of $A$.

A state on an MV-algebra $A$ is a mapping $s: A \rightarrow[0,1]$ such that $s(1)=1$ and $s(a \oplus b)=s(a)+s(b)$ whenever $a \odot b=0$. The set of all states on $A$ is denoted by $\mathcal{S}(A)$. The set $\mathcal{S}(A)$ is convex, that is, if $s_{1}, s_{2}$ are states on $A$ and $\lambda \in[0,1]$, then $\lambda s_{1}+(1-\lambda) s_{2}$ is a state on $A$. A state $s$ is extremal if it cannot be written in the form $s=\lambda s_{1}+(1-\lambda) s_{2}$, where $s_{1}, s_{2} \in \mathcal{S}(A)$ and $\lambda \in(0,1)$. The set of extremal states is denoted by $\partial_{e} \mathcal{S}(A)$. We recall that a state $s$ is extremal if and only if $\operatorname{Ker}(s)$, given by

$$
\operatorname{Ker}(s):=\{a \in A: s(a)=0\},
$$

is a maximal ideal of $A$, or equivalently, $s(a \oplus b)=\min \{s(a)+s(b), 1\}$ for all $a, b \in A$ (such a mapping is also called a state-morphism). It is possible to show that both $\mathcal{S}(A)$ and $\partial_{e} \mathcal{S}(A)$ are nonempty. When we introduce the weak topology on the set of states, that is, a net $\left\{s_{\alpha}\right\}$ of states converges weakly to a state $s$ if $\lim _{\alpha} s_{\alpha}(a)=s(a)$ for every $a \in A$, then $\mathcal{S}(A)$ and $\partial_{e} \mathcal{S}(A)$ are compact Hausdorff topological spaces. By the Krein-Mil'man theorem, [17, Theorem 5.17], every state on $A$ is a weak limit of a net of convex combinations of extremal states. In addition, the topological space $\partial_{e} \mathcal{S}(A)$ is homeomorphic to the space of all maximal ideals $\mathcal{M}(A)$ (ultrafilters $\mathcal{F}(A)$ ) with the hull-kernel topology. This homeomorphism is given by $s \leftrightarrow \operatorname{Ker}(s)$, see [11], [17, Theorem 15.32], because every maximal ideal is the kernel of a unique extremal state, and a state $s$ is extremal if and only if $\operatorname{Ker}(s)$ is a maximal ideal.

Let $A$ be an MV-algebra. An element $a \in A$ is said to be Boolean if $a \oplus a=a$. Then $a$ is Boolean if and only if any or all the following hold:

$$
a \odot a=a ; \quad a \wedge a^{*}=0 ; \quad a \vee a^{*}=1 .
$$


Let $B(A)$ be the set of all Boolean elements of $A$. Then $B(A)$ is a Boolean subalgebra of $A$. Let $a$ be a fixed Boolean element of $A$. Then the interval $[0, a]$ can be endowed with the restriction of $\oplus, \odot$ to $[0, a]$ and with ${ }^{*} a$, where $x^{* a}:=x^{*} \wedge a$ for all $x \in[0, a]$, and $\left([0, a], \oplus, \odot,^{* a}, 0, a\right)$ is an MV-algebra. The mapping $p_{a}: a \rightarrow[0, a]$ defined by $p_{a}(x)=x \wedge a$ for all $x \in A$, is an MV-homomorphism. In addition, the mapping $\Phi_{a}: a \rightarrow[0, a] \times\left[0, a^{*}\right]$, defined by

$$
\Phi_{a}(x)=\left(p_{a}(x), p_{a^{*}}(x)\right)=\left(x \wedge a, x \wedge a^{*}\right) \quad \forall x \in A,
$$

is an MV-isomorphism.

We say that an MV-algebra $A$ satisfies general comparability if, given $x, y \in A$, there is a Boolean element $a \in A$ such that $p_{a}(x) \leq p_{a}(y)$ and $p_{a^{*}}(x) \geq p_{a^{*}}(y)$. This means that the two coordinates of the elements $x=\left(p_{a}(x), p_{a^{*}}(x)\right)$ and $y=$ $\left(p_{a}(y), p_{a^{*}}(y)\right)$ can be compared in $[0, a]$ and $\left[0, a^{*}\right]$, respectively.

For example, every linearly ordered MV-algebra satisfies general comparability (trivially); every Cartesian product of linearly ordered $\mathrm{MV}$-algebras and every $\sigma$-complete MV-algebra satisfy general comparability. Further, if $A$ satisfies the general comparability, so does $A / I$ for each ideal $I$ of $A$. However, there are examples of MV-algebras that do not satisfy the general comparability.

We recall that a topological space $\Omega$ is said to be connected if it cannot be expressed as a union of two nonempty disjoint open subsets, totally disconnected if it has a base consisting of clopen (closed and open) sets, and basically disconnected provided the closure of every open $F_{\sigma}$ subset of $\Omega$ is open (an $F_{\sigma}$ set is a countable union of closed sets). Totally disconnected spaces are also called Stone spaces or Boolean spaces. For example, if $\Omega$ is finite, or if $\Omega$ is a Cantor set in $[0,1]$, then $\Omega$ is totally disconnected. Further, if $A$ is a $\sigma$-complete MV-algebra, then $\partial_{e} \mathcal{S}(A)$ is a basically disconnected, compact, Hausdorff topological space [17].

Now let $\Omega$ be a compact Hausdorff topological space and let $\mathrm{C}(\Omega)$ be the set of all continuous real-valued functions on $\Omega$. Then $\mathrm{C}(\Omega)$ is an Abelian $\ell$-group with strong unit $1_{\Omega}$ under the pointwise ordering of functions. Define the MV-algebra $C_{1}(\Omega)=\Gamma\left(\mathrm{C}(\Omega), 1_{\Omega}\right)$. Then $B\left(C_{1}(\Omega)\right)=\left\{\chi_{A}: A\right.$ is clopen in $\left.\Omega\right\}$. The system of all clopen subsets of $\Omega$ forms a Boolean algebra of a Stone space if and only if $\Omega$ is totally disconnected [17]. Therefore $C_{1}(\Omega)$ can satisfy general comparability only if $\Omega$ is totally disconnected.

For example, if $\Omega=[0,1]$ with the usual topology, then $C_{1}([0,1])$ is an MValgebra which does not satisfy general comparability, while $B\left(C_{1}([0,1])\right)=\left\{0_{\Omega}, 1_{\Omega}\right\}$. The same is true for all connected compact Hausdorff spaces $X$.

It is known that every extremal state on a Boolean algebra is two-valued. In what follows, we show every two-valued state on $B(A)$ can be uniquely extended to an extremal state on an $\mathrm{MV}$-algebra $A$ provided $A$ satisfies general comparability.

The following results concerning MV-algebras satisfying general comparability can be found in [10]. 
THEOREM 2.1. Let $A$ be an MV-algebra satisfying general comparability, and let $K$ be a maximal ideal of $B(A)$. Then there is a unique state $s$ on $A$ such that $B(A) \cap \operatorname{Ker}(s)=K$. This state is extremal.

We denote by $\mathcal{M}(B(A))$ the set of all maximal ideals of the Boolean algebra $B(A)$. With the hull-kernel topology, it is totally disconnected.

THEOREM 2.2. Let $A$ be an MV-algebra satisfying general comparability. Then the mapping,

$$
\phi(s):=B(A) \cap \operatorname{Ker}(s) \quad \forall s \in \partial_{e} \mathcal{S}(A),
$$

defines a homeomorphism $\phi$ of $\partial_{e} \mathcal{S}(A)$ onto $\mathcal{M}(B(A))$.

THEOREM 2.3. Let $A$ be an $M V$-algebra satisfying general comparability. Then $\partial_{e} \mathcal{S}(A)$ and $\partial_{e} \mathcal{S}(B(A))$ are homeomorphic compact Hausdorff totally disconnected spaces. The mapping $\phi_{A}: s \in \partial_{e} \mathcal{S}(A) \mapsto s_{\mid B(A)}$ implements the homeomorphism.

PROOF. This is a direct consequence of Theorems 2.2 and 2.1.

We recall that an extremal state $s$ is discrete if $s(A)=\{0,1 / n, \ldots, n / n\}$ for some positive integer $n$. An extremal state is discrete if and only if there exists a positive integer $n$ such that $A / \operatorname{Ker}(s)=S_{n}=: \Gamma\left(n^{-1} \mathbb{Z}, 1\right)$.

Let $A$ be an MV-algebra. Given an element $a \in A$, we define a continuous function $\hat{a}: \partial_{e} \mathcal{S}(A) \rightarrow[0,1]$ by $\hat{a}(s):=s(a)$ for all $s \in \partial_{e} \mathcal{S}(A)$. Then $\hat{A}:=\{\hat{a}: a \in A\}$ is an $\mathrm{MV}$-algebra, and the mapping,

$$
\psi(a)=\hat{a} \quad \forall a \in A,
$$

is an MV-homomorphism from $A$ onto $\hat{A}$. The mapping $\psi$ is an isomorphism if and only if $A$ is semisimple, that is, $\operatorname{Rad}(A)=\{0\}$.

The following representation of MV-algebras satisfying general comparability follows from [17, Theorem 8.20].

THEOREM 2.4. Let A be an MV-algebra satisfying general comparability. Set

$$
M(A):=\left\{f \in C_{1}\left(\partial_{e} \mathcal{S}(A)\right): f(s) \in s(A) \text { for all discrete } s \in \partial_{e} \mathcal{S}(A)\right\} .
$$

Then $\psi(A)$ is an $M V$-subalgebra of $M(A)$ that is dense in $M(A)$ in the supremum norm topology. If moreover A is semisimple, then A can be isomorphically embedded into $M(A)$.

If $A$ is a $\sigma$-complete $M V$-algebra, then $A$ is isomorphic to $M(A)$.

We note that if $A$ is an MV-algebra, then $\hat{A}$ is a subalgebra of $M(A)$.

\section{State-morphism-operators on semisimple MV-algebras}

In this section, we define state MV-algebras and we characterize state-morphismoperators, defined mainly on semisimple MV-algebras. We show that, if $A$ is representable as an MV-algebra of functions on some compact Hausdorff topological space, then each state-morphism-operator $\tau$ on $A$ is of the form $\tau(f)=f \circ g$ for all $f \in A$, for some continuous function $g: \partial_{e} \mathcal{S}(A) \rightarrow \partial_{e} \mathcal{S}(A)$ with $g^{2}=g$. 
According to $[14,15]$, a state $M V$-algebra $(A, \tau):=\left(A ; \oplus,{ }^{*}, 0, \tau\right)$ is an algebraic structure, where $\left(A ; \oplus,{ }^{*}, 0\right)$ is an MV-algebra [4] and $\tau$ is a unary operator on $A$, called an internal state or a state-operator, satisfying the following properties, for each $x, y \in A$ :

(i) $\quad \tau(0)=0$;

(ii) $\tau\left(x^{*}\right)=(\tau(x))^{*}$;

(iii) $\tau(x \oplus y)=\tau(x) \oplus \tau\left(y \odot(x \odot y)^{*}\right)$;

(iv) $\tau(\tau(x) \oplus \tau(y))=\tau(x) \oplus \tau(y)$.

In [15] it is shown that in each state MV-algebra the following hold:

- $\tau(\tau(x))=\tau(x)$;

- $\tau(1)=1$;

- $\quad$ if $x \leq y$, then $\tau(x) \leq \tau(y)$;

- $\tau(x \oplus y) \leq \tau(x) \oplus \tau(y)$;

- $\quad$ the image $\tau(A)$ is the domain of an MV-subalgebra of $A$ and $(\tau(A), \tau)$ is a state MV-subalgebra of $(A, \tau)$.

In [5], the authors defined a stronger structure, a state-morphism MV-algebra, as a state $\mathrm{MV}$-algebra $(A, \tau)$ (that is, an algebra satisfying (i)-(iv) above) with the following additional property.

(v) $\quad \tau(x \oplus y)=\tau(x) \oplus \tau(y)$.

Equivalently, $\tau$ is an MV-endomorphism of $A$ such that $\tau=\tau \circ \tau$. In this case, $\tau$ is called a state-morphism-operator.

Proposition 3.1. Let $\tau$ be a state-morphism-operator on an MV-algebra A. Then $\tau(B(A)) \subseteq B(A)$ and $\tau$ restricted to $B(A)$ is a state-morphism.

Proof. Because $\tau$ preserves $\odot$, for each Boolean element $a \in B(A)$, it is clear that $a \odot a=a$ so that $\tau(a)=\tau(a \odot a)=\tau(a) \odot \tau(a)$.

Consider the following conditions on a system $\mathcal{T}$ of functions:

(i) $1 \in \mathcal{T}$;

(ii) If $f \in \mathcal{T}$, then $1-f \in \mathcal{T}$;

(iii) if $f, g \in \mathcal{T}$, then $f \oplus g \in \mathcal{T}$, where $(f \oplus g)(\omega)=\min \{f(\omega)+g(\omega), 1\}$ for all $\omega \in \Omega$;

(iv) if $\left\{f_{n}\right\}$ is a sequence of elements of $\mathcal{T}$, then $\bigoplus_{n} f_{n} \in \mathcal{T}$, where $\left(\bigoplus_{n} f_{n}\right)(\omega):=$ $\min \left\{\sum_{n} f_{n}(\omega), 1\right\}$ for all $\omega \in \Omega$.

We say that a system $\mathcal{T}$ of functions from $[0,1]^{\Omega}$ is a Bold algebra if (i)-(iii) hold, and a tribe if $\mathcal{T}$ also satisfies (iv). Hence, every Bold algebra is an MV-algebra whilst every tribe is a $\sigma$-complete $\mathrm{MV}$-algebra, and in both cases, the MV-operations are defined pointwise.

We recall that if $A$ is an MV-algebra, then $\hat{A}:=\{\hat{a}: a \in A\}$ is a Bold algebra of continuous functions defined on the compact space $\partial_{e} \mathcal{S}(A)$. In addition, $A$ is isomorphic to $\hat{A}$ under the mapping $a \mapsto \hat{a}$ if and only if $A$ is semisimple. Then $B(A)$ 
under this representation has the form

$$
\hat{B}(A)=\{\hat{a}: a \in B(A)\}=\left\{\chi_{A}: A \text { is a clopen subset of } \partial_{e} \mathcal{S}(A)\right\} .
$$

Moreover, from Proposition 3.2, if $\tau$ is a state-operator on $A$, then we can define a state-operator $\hat{\tau}$ on $\hat{A}$ by $\hat{\tau}(\hat{a})=(\tau(a))^{\wedge}$ for all $a \in A$.

Similarly, let $\tau_{B}$ be the restriction of $\tau$ to $B(A)$ and let $\hat{\tau}_{B}$ correspond to $\tau_{B}$ defined on $\hat{B}(A)$.

Proposition 3.2. Let A be an MV-algebra, $\hat{A}$ be the associated Bold algebra, and $\tau$ be a state-morphism-operator on $A$.

(1) The mapping g that assigns to each extremal state $s \in \partial_{e} \mathcal{S}(A)$ the extremal state $s \circ \tau$ is a continuous mapping from $\partial_{e} \mathcal{S}(A)$ into itself such that $g \circ g=g$ and $g(s)(A) \subseteq s(A)$ for all discrete extremal states $s \in \partial_{e} \mathcal{S}(A)$. Let

$$
M(A)=\left\{f \in C_{1}\left(\partial_{e} \mathcal{S}(A)\right): f(s) \in s(A) \text { for all discrete } s \in \partial_{e} \mathcal{S}(A)\right\} .
$$

Define $\tau_{g}: M(A) \rightarrow M(A)$ by $\tau_{g}(f)=f \circ g$ for all $f \in M(A)$. Then $\tau_{g}$ is a state-morphism-operator on the Bold algebra $M(A)$.

(2) Define $\hat{\tau}: \hat{A} \rightarrow \hat{A}$ by $\hat{\tau}(\hat{a}):=(\tau(a))^{\wedge}$ for all $a \in A$. Then $\hat{\tau}$ is a well-defined state-morphism-operator on $\hat{A}$ that is the restriction of $\tau_{g}$.

Proof. First we prove (1). If $s$ is a state on $A$, then $s \circ \tau$ is a state on $A$ too. Further, if $s$ is extremal, then $s \circ \tau$ is extremal by the characterization of extremal states and because $\tau$ is an endomorphism. Hence the mapping $g$ on $\partial_{e} \mathcal{S}(A)$ is well defined.

Moreover, $g$ is continuous because if $s_{\alpha} \rightarrow s$, then

$$
\lim _{\alpha} g\left(s_{\alpha}\right)(a)=\lim _{\alpha} s_{\alpha}(\tau(a))=s(\tau(a))=g(s)(a) \quad \forall a \in A .
$$

From the construction of $g$ it follows that $g \circ g=g$ because

$$
g(g(s))=g(s \circ \tau)=s \circ \tau \circ \tau=s \circ \tau=g(s) \quad \forall s \in \partial_{e} \mathcal{S}(A) .
$$

Let $s$ be a discrete state on $A$. Then $s(A)=\{0,1 / n, \ldots, n / n\}$ for some positive integer $n$. Then $s(\tau(A)) \subseteq\{0,1 / n, \ldots, n / n\}$, and because $g(s)$ is an extremal state, $s(\tau(A))=\{0,1 / m, \ldots, m / m\}$ for some divisor $m$ of $n$.

Now take $f \in M(A)$. Then $f$ is a continuous function taking values in the interval $[0,1]$. To verify that $\tau_{g}(f) \in M(A)$ we have to show that $\tau_{g}(f)(s) \in s(A)$ for all discrete extremal states $s$ on $A$. We can check that

$$
\tau_{g}(f)(s)=f(g(s))=f(s \circ \tau) \in(s \circ \tau)(A) \subseteq s(A)
$$

by the statement just proved. Hence, $\tau_{g}(f)$ is also an element of $M(A)$. It is now easy to verify that $\tau_{g}$ is a state-morphism-operator on the Bold algebra $M(A)$.

Now we prove (2). We are going to show that $\hat{\tau}$ is a well-defined operator on $\hat{A}$. Assume that $\hat{a}=\hat{b}$. This means that $s(a)=s(b)$ for all $s \in \partial_{e} \mathcal{S}(A)$. Hence

$$
s(\tau(a))=g(s)(a)=g(s)(b)=s(\tau(b)),
$$

so that $(\tau(a))^{\wedge}=(\tau(b))^{\wedge}$ and then $\hat{\tau}(\hat{a})=\hat{a} \circ g=\hat{b} \circ g=\hat{\tau}(\hat{b})$. Now $\hat{A}$ is a subalgebra of $M(A)$, so $\hat{\tau}$ is the restriction of $\tau_{g}$. 
REMARK 3.3. We summarize Proposition 3.2: if $A$ is a semisimple MV-algebra and $\tau$ is a state-morphism-operator on $A$, then $\tau$ is uniquely determined by an appropriate continuous function $g$.

THEOREM 3.4. Let $\tau$ be a state-morphism-operator on an $M V$-algebra A. Then there is a continuous function $g: \partial_{e} \mathcal{S}(A) \rightarrow \partial_{e} \mathcal{S}(A)$ such that $g \circ g=g, g(s)(A) \subseteq s(A)$ for all discrete extremal states $s$ and $(\tau(a))^{\wedge}=\hat{\tau}(\hat{a})=\hat{a} \circ g$ for all $a \in A$.

Proof. Take the continuous function $g$ defined in Proposition 3.2. Then $g \circ g=g$ and $g(s)(A) \subseteq s(A)$ for all discrete extremal states $s$ on $A$. Define $\hat{\tau}$ on $\hat{A}$ by $\hat{\tau}(\hat{a})=(\tau(a))^{\wedge}$ for all $a \in A$, as in Proposition 3.2. Then $\hat{\tau}$ is a state-morphismoperator on $\hat{A}$. Now let $s \in \partial_{e} \mathcal{S}(A)$ and $a \in A$. Then

$$
\hat{\tau}(\hat{a})(s)=\tau(\hat{a})(s)=s(\tau(a))=(s \circ g)(a)=g(s)(a)=\hat{a}(g(s))=(\hat{a} \circ g)(s),
$$

as required.

Let $B$ be a Boolean algebra and let $\partial_{e} \mathcal{S}(B)$ be the system of all extremal states on $B$. Then each such state is two-valued on $B$. For each $b \in B$, define the continuous function $\hat{b}$ on $\partial_{e} \mathcal{S}(B)$ by $\hat{b}(s)=s(b)$ for all $s \in \partial_{e} \mathcal{S}(B)$, and let $\hat{B}:=\{\hat{b}: b \in B\}$. Each $\hat{b}$ is in fact the characteristic function of some clopen set $E \subseteq \partial_{e} \mathcal{S}(B)$. Let $\tau_{B}$ be a state-operator on $B$, and let $g=g_{B}$ be the continuous function on $\partial_{e} \mathcal{S}(B)$ whose existence is guaranteed by Proposition 3.2.

Proposition 3.5. Let $B$ be a Boolean algebra and $\tau_{B}$ be a state-operator on $B$. Then $\tau_{B}$ is a state-morphism-operator. Define the mapping $\tau_{g_{B}}$ on $\hat{B}$ by $\tau_{g_{B}}(\hat{b})=$ $\hat{b} \circ g_{B}$ for all $\hat{b} \in \hat{B}$. Then $\tau_{g_{B}}$ is a state-morphism on $\hat{B}$ and $\tau_{g_{B}}=\hat{\tau}_{B}$, where $\hat{\tau}_{B}$ is the state-morphism-operator on $\hat{B}$ defined by $\hat{\tau}_{B}(\hat{b})=\tau_{B}(b)^{\wedge}$.

Proof. Let $s \in \partial_{e} \mathcal{S}(B)$. Then we have $\tau_{g_{B}}(\hat{b})(s)=\hat{b}(g(s))=\hat{b}\left(s \circ \tau_{B}\right)=s\left(\tau_{B}(b)\right)=$ $\hat{\tau}_{B}(\hat{b})(s)$.

Proposition 3.6. Suppose that $A$ is a $\sigma$-complete $M V$-algebra. Then the mapping $\psi: A \rightarrow C_{1}\left(\partial_{e} \mathcal{S}(A)\right)$, given by $\psi(a)=\hat{a}$ for all $a \in A$, preserves all countable suprema and infima that exist in $A$.

Proof. If $A=\Gamma(G, u)$, where $(G, u)$ is an Abelian $\ell$-group with strong unit $u$, then, by [18], $A$ is $\sigma$-complete if and only if $G$ is Dedekind $\sigma$-complete, that is, if $g_{n}, g \in G$ and $g_{n} \leq g$ for all $n \geq 1$ imply that $\bigvee_{n} g_{n}$ exists in $G$. Applying the Mundici functor [4] and [17, Lemma 9.12], we have the desired statement.

Let $A$ be an MV-algebra. We introduce a partial binary operation + as follows: $a+b$ is defined in $A$ if and only if $a \leq b^{*}$ and, when it is defined, $a+b:=a \oplus b$. Then the operation + is commutative and associative. Further, if $A=\Gamma(G, u)$, then $a+b$ corresponds to the group addition + in the Abelian $\ell$-group $G$.

We define $0 \cdot a:=0$ and $1 \cdot a:=a$. Inductively, if $n \cdot a$ is defined in $A$ and $n \cdot a \leq a^{*}$, then we set $(n+1) \cdot a:=(n \cdot a)+a$. Now $\operatorname{Rad}(A)$ consists of all elements $a \in A$ such that $n \cdot a$ exists in $A$ for each integer $n \geq 1$. Such elements are said to be infinitesimal. 
We say that a state-operator $\tau$ on an MV-algebra $A$ is monotone $\sigma$-complete if $a_{n} \nearrow a$ (that is, $a_{n} \leq a_{n+1}$ for all $n \geq 1$ and $\left.a=\bigvee_{n} a_{n}\right)$ implies that $\tau(a)=\bigvee_{n} \tau\left(a_{n}\right)$. We recall that if $\tau$ is monotone $\sigma$-complete, then it preserves all countable suprema and infima that exist in $A$, and we call it a $\sigma$-complete state-morphism-operator.

For any function $f: \partial_{e} \mathcal{S}(A) \rightarrow[0,1]$, we set $N(f):=\left\{s \in \partial_{e} \mathcal{S}(A): f(s) \neq 0\right\}$.

THEOREM 3.7. Let $\tau$ be a $\sigma$-complete state-morphism-operator on a $\sigma$-complete $M V$-algebra $A$. Then there is a continuous function $g$ defined on $\partial_{e} \mathcal{S}(A)$ such that $g \circ g=g, g(s)(A) \subseteq s(A)$ for all discrete extremal states $s$ on $E$ and $\hat{\tau}(\hat{a})=\hat{a} \circ g$ for all $a \in A$.

Conversely, let $g: \partial_{e} \mathcal{S}(A) \rightarrow \partial_{e} \mathcal{S}(A)$ be a continuous function such that $g \circ g=g$ and $g(s)(A) \subseteq s(A)$ for all discrete extremal states $s$. Define the mapping $\tau_{g}$ on $\hat{A}$ by $\tau_{g}(\hat{a}):=\hat{a} \circ g$ for all $a \in A$. Then $\tau_{g}$ is a $\sigma$-complete state-morphism-operator on $\hat{A}$.

In addition, if $\tilde{\tau}_{g}$ is defined on $A$ via $\tilde{\tau}_{g}(a)=\tau_{g}(\hat{a})$ for all $a \in A$, then $\tilde{\tau}_{g}$ is a $\sigma$ complete state-morphism-operator on $A$, and $g(s)=s \circ \tilde{\tau}_{g}$ for all $s \in \partial_{e} \mathcal{S}(A)$.

ProOf. Since $A$ is necessarily semisimple, because $A$ is $\sigma$-complete, it follows from Theorem 3.4 that $(\tau(a))^{\wedge}=\hat{\tau}(\hat{a})=\hat{a} \circ g$ for all $a \in A$.

By Proposition 3.2(2), the mapping $\tau_{g}$, defined on $\hat{A}$ by $\tau_{g}(\hat{a}):=\hat{a} \circ g$ for all $a \in A$, is a state-morphism-operator on $\hat{A}$.

Assume that $a_{n} \nearrow a$. Then $\hat{a}_{n} \circ g \leq \hat{a}_{n+1} \circ g \leq \hat{a} \circ g$. Further, $a=\bigvee_{n} a_{n}$, whence $\hat{a}=\bigvee_{n} \hat{a}_{n}$.

If $a_{0}(s)=\lim _{n} \hat{a}_{n}(s)$ for all $s \in \partial_{e} \mathcal{S}(A)$, that is, $a_{0}$ is a pointwise limit of a sequence of continuous functions on a compact Hausdorff space, then by [21, pp. 86, 405-406], the set $N\left(a_{0}-\hat{a}\right)$ is meager. Similarly, $N\left(\hat{a} \circ g-a_{0} \circ g\right)$ is a meager set. If $h=\bigvee_{n} \hat{a}_{n} \circ g$, then $h \leq \hat{a} \circ g$. Since

$$
N(h-\hat{a} \circ g) \subseteq N\left(h-a_{0} \circ g\right) \cup N\left(a_{0} \circ g-\hat{a} \circ g\right),
$$

it follows that $N(h-\hat{a} \circ g)$ is a meager set. By the Baire category theorem, no nonempty open subset of a compact Hausdorff space can be meager, and consequently $N(h-\hat{a} \circ g)=\emptyset$, that is, $h=\hat{a} \circ g$.

Finally, let $a \in A$ and $s \in \partial_{e} \mathcal{S}(A)$. Then

$$
\left(s \circ \tilde{\tau}_{g}\right)(a)=s\left(\tilde{\tau}_{g}(a)\right)=s\left(\tau_{g}(\hat{a})\right)=\hat{a}(g(s))=g(s)(a),
$$

that is, $g(s)=s \circ \tilde{\tau}_{g}$ for all $s \in \partial_{e} \mathcal{S}(A)$.

Two alternative proofs for special cases of Theorem 3.7 are presented in Section 5.

\section{The Loomis-Sikorski theorem}

We now present the main result of this paper: a generalization of the LoomisSikorski theorem for $\sigma$-complete state-morphism MV-algebras. We show that each such algebra is a $\sigma$-epimorphic image of some tribe, that is, a $\sigma$-complete $\mathrm{MV}$-algebra of functions on some nonempty set $\Omega$, where the $\mathrm{MV}$-operations are defined pointwise, 
and the state-morphism-operator is induced by an idempotent function $g$. In addition, we present a continuous version of the Loomis-Sikorski theorem.

Let $A$ be a $\sigma$-complete MV-algebra. Then $\hat{A}=M(A)$, but $\hat{A}$ is not necessarily a tribe. Let $\mathcal{T}(A)$ be the tribe of functions on $[0,1]^{\partial_{e} \mathcal{S}(A)}$ generated by $\hat{A}=M(A)$.

Proposition 4.1. Let $A$ be a $\sigma$-complete $M V$-algebra and let $g$ be a continuous function on $\partial_{e} \mathcal{S}(A)$ such that $g \circ g=g$ and $g(s)(A) \subseteq s(A)$ for all discrete $s \in$ $\partial_{e} \mathcal{S}(A)$. Then the operator $\mathcal{T}_{g}$, defined on $\mathcal{T}(A)$ by $\mathcal{T}_{g}(f)=f \circ g$ for all $f \in \mathcal{T}(A)$, is a $\sigma$-complete state-morphism-operator that is the unique extension of the $\sigma$-complete state-morphism-operator $\tau_{g}$ on $M(A)$ defined by $\tau_{g}(f)=f \circ g$ for all $f \in M(A)$.

PROOF. First, we show that $\mathcal{T}_{g}$ is a well-defined operator on $\mathcal{T}(A)$, that is, if $f \in$ $\mathcal{T}(A)$, then $f \circ g \in \mathcal{T}(A)$. Let $\mathcal{T}^{\prime}$ be the set of all $f \in \mathcal{T}(A)$ such that $f \circ g \in \mathcal{T}(A)$. Then $\mathcal{T}^{\prime}$ contains $M(A)=\hat{A}$ and if $f \in \mathcal{T}^{\prime}$, then $1-f \in \mathcal{T}^{\prime}$. Now let $f_{1}, f_{2} \in \mathcal{T}^{\prime}$, then $f_{1} \oplus f_{2}$ and $f_{1} \vee f_{2}$ belong to $\mathcal{T}^{\prime}$. Hence, if $\left\{f_{n}\right\}$ is a sequence of monotone functions from $\mathcal{T}^{\prime}$, then $f \circ g=\lim _{n} f_{n} \circ g \in \mathcal{T}^{\prime}$, where $f=\lim _{n} f_{n}$. This implies that $\mathcal{T}^{\prime}$ is the tribe generated by $M(A)$, and consequently, $\mathcal{T}^{\prime}=\mathcal{T}(A)$ and $\mathcal{T}_{g}$ is a $\sigma$-complete state-morphism-operator on $\mathcal{T}(A)$ that is an extension of $\tau_{g}$.

Now, if $\tau$ is any $\sigma$-complete state-morphism-operator on $\mathcal{T}(A)$ that is an extension of $\tau_{g}$, then the set of elements $f \in \mathcal{T}(A)$ such that $\tau(f)=\mathcal{T}_{g}(f)$ is a tribe containing $M(A)$, and so has to be $\mathcal{T}(A)$, whence $\tau=\mathcal{T}_{g}$.

We now characterize the tribe generated by $C_{1}(\Omega)=\Gamma\left(\mathrm{C}(\Omega), 1_{\Omega}\right)$, where $\mathrm{C}(\Omega)$ is the space of all continuous fuzzy functions on a compact Hausdorff space $\Omega$. We recall that $\mathcal{B}(\Omega)$ denotes the Baire $\sigma$-algebra generated by compact $G_{\delta}$ sets on $\Omega$ (a $G_{\delta}$ set is a countable intersection of open sets), or equivalently, by the collection $\left\{f^{-1}([a, \infty)): f \in \mathrm{C}(\Omega), a \in \mathbb{R}\right\}$.

The following result can be found, for example, in [12, Proposition 7.1.11].

Proposition 4.2. Let $\Omega$ be a compact Hausdorff space. Then $\mathcal{T}\left(C_{1}(\Omega)\right)=\mathcal{M}(\Omega)$, where $\mathcal{T}\left(C_{1}(\Omega)\right)$ is the tribe generated by $C_{1}(\Omega)$, and $\mathcal{M}(\Omega)$ is the set of all Baire measurable functions on $[0,1]^{\Omega}$.

PROpOSITION 4.3. Let $\mathcal{T}$ be a tribe of functions defined on a nonempty set $\Omega$. Let $g$ be a function on $\Omega$ such that $g \circ g=g$ and $f \circ g \in \mathcal{T}$ for all $f \in \mathcal{T}$. Then the operator $\tau_{g}: \mathcal{T} \rightarrow \mathcal{T}$, defined by $\tau_{g}(f)=f \circ g$ for all $f \in \mathcal{T}$, is a $\sigma$-complete state-morphismoperator.

Proof. Clearly $\tau_{g}$ is a state-morphism-operator on $\mathcal{T}$. Suppose that $f_{n}(\omega) \nearrow f(\omega)$ for all $\omega \in \Omega$. Then $f_{n}(g(\omega)) \nearrow f(g(\omega))$ for all $\omega \in \Omega$, so that $\tau_{g}$ is monotone $\sigma$-complete; consequently, it is a $\sigma$-complete state-morphism-operator.

Suppose that $\left(A_{1}, \tau_{1}\right)$ and $\left(A_{2}, \tau_{2}\right)$ are state MV-algebras. An MV-homomorphism $h: A_{1} \rightarrow A_{2}$ is said to be a state MV-homomorphism if $h \circ \tau_{1}=\tau_{2} \circ h$. Similarly we define both a state-morphism $M V$-homomorphism if $\tau_{1}$ and $\tau_{2}$ are state-morphisms, and a $\sigma$-state-morphism $M V$-homomorphism if $\left(A_{1}, \tau_{1}\right)$ and $\left(A_{2}, \tau_{2}\right)$ are $\sigma$-complete state-morphism MV-algebras and $h$ is a state-morphism $\sigma$-MV-homomorphism. 
We now present a variant of the Loomis-Sikorski theorem for $\sigma$-complete statemorphism MV-algebras.

THEOREM 4.4 (Loomis-Sikorski theorem). Let $(A, \tau)$ be a $\sigma$-complete statemorphism MV-algebra. Then there are a $\sigma$-complete state-morphism $M V$-algebra $\left(\mathcal{T}, \mathcal{T}_{g}\right)$, where $\mathcal{T}$ is a tribe of functions from $[0,1]^{\Omega}$ and a function $g: \Omega \rightarrow \Omega$ such that $g \circ g=g$ and $f \circ g \in \mathcal{T}$ for all $f \in \mathcal{T}$, such that $\mathcal{T}_{g}$, defined by $\mathcal{T}_{g}(f):=f \circ g$ for all $f \in \mathcal{T}$, is a $\sigma$-complete state-morphism-operator on $\mathcal{T}$. Moreover, there is a $\sigma$-state-morphism $M V$-homomorphism $h$ from $\mathcal{T}$ onto A such that $h \circ \mathcal{T}_{g}=\tau \circ h$.

PROOF. Let $A$ be a $\sigma$-complete MV-algebra with a $\sigma$-complete state-morphismoperator $\tau$. We isomorphically embed $A$ onto $\hat{A}$. We set $\Omega=\partial_{e} \mathcal{S}(A)$; then $\Omega$ is a basically disconnected compact Hausdorff topological space and $\hat{A}=M(A)$. Let $\mathcal{T}=\mathcal{T}(A)$ be the tribe of functions from $[0,1]^{\Omega}$ that is generated by $\hat{A}$. According to Proposition 3.2, the function $g: \partial_{e} \mathcal{S}(A) \rightarrow \partial_{e} \mathcal{S}(A)$, defined by $g(s)=s \circ g$ for all $s \in \partial_{e} \mathcal{S}(A)$, is continuous and $g \circ g=g$. The mapping $\mathcal{T}_{g}: \mathcal{T} \rightarrow \mathcal{T}$, defined by $\mathcal{T}_{g}(f)=f \circ g$ for all $f \in \mathcal{T}(A)$, is a $\sigma$-complete state-morphism-operator on $\mathcal{T}$ by Theorem 3.7, and by Proposition 4.1, it is a unique extension of the $\sigma$-complete statemorphism-operator $\tau_{g}$ on $\mathcal{T}$, defined by $\tau_{g}(\hat{a})=\hat{a} \circ g$ for all $a \in A$.

Let $f \in \mathcal{T}$ and $a \in A$. We will say that $f \sim a$ if $N(f-\hat{a}):=\left\{s \in \partial_{e} \mathcal{S}(A)\right.$ : $f(s) \neq \hat{a}(s)\}$ is a meager set. Let us denote by $\mathcal{T}^{\prime}$ the set of all functions $f \in \mathcal{T}$ such that there is $a \in A$ with $f \sim a$.

If $a_{1}$ and $a_{2}$ are two elements of $A$ such that $f \sim a_{1}$ and $f \sim a_{2}$, then

$$
N\left(\hat{a}_{1}-\hat{a}_{2}\right) \subseteq N\left(f-\hat{a}_{1}\right) \cup N\left(f-\hat{a}_{2}\right),
$$

so $N\left(\hat{a}_{1}-\hat{a}_{2}\right)$ is a meager set. The functions $\hat{a}_{1}$ and $\hat{a}_{2}$ are continuous, and it follows from the Baire category theorem that $\hat{a}_{1}=\hat{a}_{2}$.

Therefore the mapping $h: \mathcal{T}^{\prime} \rightarrow A$ defined by $h(f)=a$ when $f \sim a$ is well defined. In [9], it was proved that $\mathcal{T}^{\prime}$ is a tribe containing $\hat{A}$, so $\mathcal{T}^{\prime}=\mathcal{T}$, and $h$ is in fact a $\sigma$-homomorphism from $\mathcal{T}$ onto $A$.

Finally, we now let $f \in \mathcal{T}$ and $a \in A$ be such $h(f)=a$. Then $f \sim a$ so that $N(f-\hat{a})$ is a meager set. Then $N(f \circ g-\hat{a} \circ g)=g^{-1}(N(f-\hat{a}))$ is also meager. It follows from Theorem 3.4 that $h\left(\mathcal{T}_{g} f\right)=\tau(a)=\tau(h(f))$.

Theorem 4.4 can also be reformulated using topological language.

THEOREM 4.5. Let $(A, \tau)$ be a $\sigma$-complete state-morphism MV-algebra. Then there is a nonempty basically disconnected compact Hausdorff topological space $\Omega$, a tribe $\mathcal{T}$ of functions on $[0,1]^{\Omega}$, and a continuous function $g: \Omega \rightarrow \Omega$ such that $g \circ g=g$ and $f \circ g \in \mathcal{T}$ for all $f \in \mathcal{T}$, such that $\mathcal{T}_{g}$, given by $\mathcal{T}_{g}(f):=f \circ g$ for all $f \in \mathcal{T}$, is a $\sigma$-complete state-morphism-operator on $\mathcal{T}$. Moreover, there is a $\sigma$-homomorphism $h$ from $\mathcal{T}$ onto A such that $h \circ \mathcal{T}_{g}=\tau \circ h$.

PROOF. Set $\Omega=\partial_{e} \mathcal{S}(A)$; then the result follows from the proof of Theorem 4.4.

Let $(A, \tau)$ be a $\sigma$-complete state-morphism MV-algebra. We define a quintuple $\left(\Omega, \mathcal{T}, g, \mathcal{T}_{g}, h\right)$, where $\Omega=\partial_{e} \mathcal{S}(A), \mathcal{T}=\mathcal{T}(A), g$ is the continuous function on $\Omega$ 
such that $g \circ g=g$ defined by Proposition 3.2, $\mathcal{T}_{g}(f)$, given by $\mathcal{T}_{g}(f)=f \circ g$ for all $f \in \mathcal{T}$, is a $\sigma$-complete state-morphism on $\mathcal{T}$, and $h$ is a $\sigma$-MV-homomorphism from $\mathcal{T}$ onto $A$ such that $h \circ \mathcal{T}_{g}=\tau \circ h$. Then $\left(\Omega, \mathcal{T}, g, \mathcal{T}_{g}, h\right)$ is said to be a canonical representation of the $\sigma$-complete state-morphism MV-algebra $(A, \tau)$.

\section{Alternative proofs for special cases}

In this final section, we give alternative proofs to Theorem 3.7 for two special cases: for tribes, in Theorem 5.2, and for weakly divisible $\sigma$-complete MV-algebras, in Theorem 5.4.

First, the following result can be found, for example, in [12, Theorem 7.1.7].

THEOREM 5.1. Let $\mathcal{T}$ be a tribe of $[0,1]$-valued functions on the nonempty set $\Omega$, and define

$$
\mathcal{S}_{0}(\mathcal{T}):=\left\{A \subseteq \Omega: \chi_{A} \in \mathcal{T}\right\} .
$$

Then the following results hold.

(1) $\mathcal{S}_{0}(\mathcal{T})$ is a $\sigma$-algebra of subsets of $\Omega$.

(2) If $f \in \mathcal{T}$, then $f$ is $\mathcal{S}_{0}(\mathcal{T})$-measurable.

(3) $\mathcal{T}$ contains all $\mathcal{S}_{0}(\mathcal{T})$-measurable functions from $\Omega$ into the real interval $[0,1]$ if and only if $\mathcal{T}$ contains all constant functions with values in $[0,1]$.

THEOREM 5.2. Let $\mathcal{T}$ be a tribe of functions from $[0,1]^{\Omega}$ containing all constant functions and let $\tau$ be a $\sigma$-complete state-morphism-operator on $\mathcal{T}$ such that the tribe $\mathcal{T}$ is countably generated and such that $\chi_{\{\omega\}} \in \mathcal{T}$ for all $\omega \in \Omega$. Then there is a unique $\mathcal{S}_{0}(\mathcal{T})$-measurable function $g$ from $\Omega$ into itself such that $g \circ g=g$ and $\tau_{g}=\tau$, where $\tau_{g}(f):=f \circ g$ for all $f \in \mathcal{T}$.

PROOF. There are three steps in the proof.

Step 1. Note that $\mathcal{T}$ is countably generated if and only if $\mathcal{S}_{0}(\mathcal{T})$ is countably generated, where $\mathcal{S}_{0}(\mathcal{T})$ is defined by (5.1). Indeed, if $\left\{f_{n}\right\}$ is a countable generator of $\mathcal{T}$, then $\left\{f_{n}^{-1}(B): B \in \mathcal{B}_{0}\right\}$, where $\mathcal{B}_{0}$ is a countable generator of the Borel $\sigma$-algebra $\mathcal{B}(\mathbb{R})$, is a countable generator of $\mathcal{S}_{0}(\mathcal{T})$.

Conversely, let $\left\{A_{n}\right\}$ be a countable generator of $\mathcal{S}_{0}(\mathcal{T})$. We assert that the system $\left\{r_{n} \chi_{A_{n}}\right\}$, where each $r_{n}$ is a rational number in [0,1], is a countable generator of $\mathcal{T}$. Let $\mathcal{T}^{\prime}$ be the tribe generated by $\left\{r_{n} \chi_{A_{n}}\right\}$. Then $\chi_{A} \in \mathcal{T}^{\prime}$ for all $A \in \mathcal{S}_{0}(\mathcal{T})$, so $t \chi_{A} \in \mathcal{T}^{\prime}$ for all $t \in[0,1]$ and $A \in \mathcal{S}_{0}(\mathcal{T})$. Therefore every step function $f=\sum_{i=1}^{k} t_{i} \chi_{B_{i}}$, where $t_{i} \in[0,1]$ and $B_{1}, \ldots, B_{k}$ are mutually disjoint sets from $\mathcal{S}_{0}(\mathcal{T})$, is in $\mathcal{T}^{\prime}$. It is well known that if $f \in \mathcal{T}$, then there is a sequence of step functions $\left\{f_{n}\right\}$ in $\mathcal{T}^{\prime}$ such that $f_{n} \nearrow f$, and this implies that $f \in \mathcal{T}^{\prime}$. Hence, $\mathcal{T}^{\prime}=\mathcal{T}$.

Step 2. Given $\omega \in \Omega$, let $I_{\omega}:=\{f \in \mathcal{T}: f(\omega)=0\}$. This is a $\sigma$-ideal of $\mathcal{T}$, that is, if $f_{n} \in I_{\omega}$ then $\sup _{n} f_{n} \in I_{\omega}$. If $f \in \mathcal{T} \backslash I_{\omega}$, then $f(\omega)>0$, so there is a positive integer $n$ such that $n f(\omega) \wedge 1=1$, whence $(n f)^{*} \in I_{\omega}$, and this says that $I_{\omega}$ is a maximal ideal. 
Conversely, let $I$ be any maximal ideal of $\mathcal{T}$ that is a $\sigma$-ideal. We claim that there is a unique $\omega \in \Omega$ such that $I=I_{\omega}$. Let $\hat{I}:=\left\{A \in \mathcal{S}_{0}(\mathcal{T}): \chi_{A} \in I\right\}$. Then $\hat{I}$ is a maximal ideal of $\mathcal{S}_{0}(\mathcal{T})$ that is also a $\sigma$-ideal, that is, if $C_{n} \in \hat{I}$ when $n \geq 1$, then $\bigcup_{n} C_{n} \in \hat{I}$. Since if $\left\{A_{n}\right\}$ is a generator of $\mathcal{S}_{0}(\mathcal{T})$, then $\left\{B_{n}\right\}$, where $B_{n}=A_{n}$ if $\omega \notin A_{n}$ and $B_{n}=$ $\Omega \backslash A_{n}$ otherwise, is also a generator of $\mathcal{S}_{0}(\mathcal{T})$. Set $B_{0}=\bigcap_{n} B_{n}$, then $B_{0} \in \mathcal{S}_{0}(\mathcal{T})$. Let $\mathcal{S}_{0}:=\left\{A \in \mathcal{S}_{0}(\mathcal{T}): a \cap B_{0}=\emptyset\right.$ or $\left.A \supseteq B_{0}\right\}$. Then $\mathcal{S}_{0}$ is a $\sigma$-algebra containing the generator $\left\{B_{n}\right\}$ so that $\mathcal{S}_{0}=\mathcal{S}_{0}(\mathcal{T})$. Since each singleton $\{\omega\}$ belongs to $\mathcal{S}_{0}$, then there is a unique $\omega \in \Omega$ such that $B_{0}=\{\omega\}$. Now let $I_{\omega}^{\prime}:=\{f \in I: f(\omega)=0\}$. Then each $\chi_{B_{n}} \in I_{\omega}^{\prime}$ as well as $\chi_{A} \in I_{\omega}^{\prime}$ whenever $A \in \hat{I}$. Because $t \chi_{A} \leq \chi_{A}$, we have $t \chi_{A} \in I_{\omega}^{\prime}$ and therefore each step function $f=\sum_{i=1}^{k} t_{i} \chi_{C_{i}} \in I_{\omega}^{\prime}$ with $t_{i} \in[0,1]$ and with mutually disjoint sets $C_{1}, \ldots, C_{k} \in \hat{I}$. Hence, by approximating any function $f \in I$ from below by step functions from $I_{\omega}^{\prime}$, we see that $f \in I_{\omega}^{\prime}$ and $I=I_{\omega}$.

Step 3. Let $I_{\omega}$ be given. Then $\tau^{-1}\left(I_{\omega}\right):=\left\{f \in \mathcal{T}: \tau(f) \in I_{\omega}\right\}$ is also a maximal ideal that is a $\sigma$-ideal. By Step 2, there is a unique $\omega^{\prime} \in \Omega$ such that $\tau^{-1}\left(I_{\omega}\right)=I_{\omega^{\prime}}$, so we can define a function $g: \Omega \rightarrow \Omega$ such that $g(\omega)=\omega^{\prime}$ if and only if $\tau^{-1}\left(I_{\omega}\right)=I_{\omega^{\prime}}$. It is clear that $g \circ g=g$.

Given $\omega \in \Omega$, define $s_{\omega}: \mathcal{T} \rightarrow[0,1]$ by $s_{\omega}(f):=f(\omega)$ for all $f \in \mathcal{T}$. Then $s_{\omega}$ is an extremal state that is $\sigma$-continuous, that is, if $f_{n} \nearrow f$, then $s_{\omega}(f)=\lim _{n} s_{\omega}\left(f_{n}\right)$. Then $\operatorname{Ker}\left(s_{\omega}\right)=I_{\omega}$ and $f=g$ if and only if $s_{\omega}(f)=s_{\omega}(g)$ for all $\omega \in \Omega$. Moreover,

$$
\operatorname{Ker}\left(s_{\omega} \circ \tau\right)=\left(s_{\omega} \circ \tau\right)^{-1}(\{0\})=\tau^{-1}\left(I_{\omega}\right)=I_{g(\omega)} .
$$

Then

$$
(\tau(f))(\omega)=s_{\omega}(\tau(f))=s_{\omega} \circ \tau \circ f=s_{g(\omega)} \circ f=f(g(\omega)),
$$

and so $\tau(f)=f \circ g \in \mathcal{T}$ for all $f \in \mathcal{T}$. We show that $g$ is $\mathcal{S}_{0}(\mathcal{T})$-measurable. For all $B \in \mathcal{B}_{0}(\mathbb{R})$,

$$
(\tau(f))^{-1}(B)=(f \circ g)^{-1}(B)=g^{-1}\left(f^{-1}(B)\right) \in \mathcal{S}_{0}(\mathcal{T}) .
$$

Hence, if $A \in \mathcal{S}_{0}(\mathcal{T})$ and $B=\{1\}$, then $g^{-1}(A)=g^{-1}\left(\chi_{A}^{-1}(\{1\})\right) \in \mathcal{S}_{0}(\mathcal{T})$, so $g$ is $\mathcal{S}_{0}(\mathcal{T})$-measurable.

Hence, the mapping $\tau_{g}$, defined by $\tau_{g}(f):=f \circ g$ for all $f \in \mathcal{T}$, is a $\sigma$-complete state-morphism-operator on $\mathcal{T}$ such that $\tau=\tau_{g}$. Now let $g^{\prime}: \Omega \rightarrow \Omega$ be an $\mathcal{S}_{0}(T)$ measurable function such that $g^{\prime} \circ g^{\prime}=g^{\prime}$ and $f \circ g^{\prime}=f \circ g$ for all $f \in \mathcal{T}$. Then for all $A \in \mathcal{S}_{0}(T)$, we have $\chi_{A} \circ g^{\prime}=\chi_{A} \circ g$, that is, $g^{-1}(A)=g^{-1}(A)$. If $\omega_{0}$ is an element of $\Omega$, then $\left\{\omega \in \Omega: g^{\prime}(\omega)=\omega_{0}\right\}=\left\{\omega \in \Omega: g(\omega)=\omega_{0}\right\}$. As $\omega_{0}$ is arbitrary, this yields $g^{\prime}=g$.

The second case depends on the notions of divisibility and the following lemma.

LEMMA 5.3. Let $\Omega$ be a basically disconnected compact Hausdorff topological space. For each continuous function $f: \Omega \rightarrow[0,1]$, there is a monotone sequence $\left\{f_{n}\right\}$ of continuous step functions defined on $\Omega$ and with values in the interval $[0,1]$ such that $f_{n} \nearrow f$ uniformly. 
PROOF. There are three steps in the proof.

Step 1. Let $X$ be a clopen subset of $\Omega$ and $f: X \rightarrow[\alpha, \beta]$ be a continuous function, where $0 \leq \alpha<\beta \leq 1$. Then there are two mutually disjoint clopen sets $X_{1}$ and $X_{2}$ such that $X=X_{1} \cup X_{2}$ and $f\left(X_{1}\right) \subseteq[\alpha,(\alpha+\beta) / 2]$ and $f\left(X_{2}\right) \subseteq[(\alpha+\beta) / 2, \beta]$. Indeed, the set $f^{-1}(((\alpha+\beta) / 2, \beta])$ is an open $F_{\sigma}$ set. Its closure $X_{2}$ is both open and closed. Then $X_{1}=X \backslash X_{2}$ is also a clopen set, $f\left(X_{1}\right) \subseteq[\alpha,(\alpha+\beta) / 2]$ and $f\left(X_{2}\right) \subseteq[(\alpha+\beta) / 2, \beta]$.

The function $g: X \rightarrow[\alpha, \beta]$, defined by $g(x)=\alpha$ if $x \in X_{1}$ and $g(x)=(\alpha+\beta) / 2$ if $x \in X_{2}$, is continuous, $g \leq f$ and $f(x)-g(x) \leq(\beta-\alpha) / 2$ for all $x \in X$.

Step 2. Let $f: \Omega \rightarrow[0,1]$ be a continuous function. Setting $X_{0}=\Omega$ and applying Step 1, we can find two disjoint clopen sets $X_{1}^{1}$ and $X_{2}^{1}$ such that $X_{0}=X_{1}^{1} \cup X_{2}^{1}$ and $f\left(X_{1}^{1}\right) \subseteq[0,1 / 2]$ and $f\left(X_{1}^{2}\right) \subseteq[1 / 2,1]$.

Suppose inductively that we have partitioned $X$ into mutually disjoint clopen sets $X_{n}^{0}, X_{n}^{1}, \ldots, X_{n}^{2^{n}-1}$ such that $f\left(X_{n}^{i}\right) \subseteq\left[i / 2^{n},(i+1) / 2^{n}\right]$ when $i=0,1, \ldots$, $2^{n}-1$.

Using Step 1, we decompose each of the sets $X_{n}^{0}, X_{n}^{1}, \ldots, X_{n}^{2^{n}-1}$ into two mutually disjoint clopen sets, to obtain a partition of $X$ into clopen sets $X_{n+1}^{0}, X_{n+1}^{1}, \ldots, X_{n+1}^{2^{n+1}-1}$ such that $f\left(X_{n+1}^{i}\right) \subseteq\left[i / 2^{n+1},(i+1) / 2^{n+1}\right]$ when $i=$ $0,1, \ldots, 2^{n+1}-1$.

Step 3. Given a sequence of refining partitions into clopen sets $X_{n}^{0}, X_{n}^{1}, \ldots, X_{n}^{2^{n}-1}$, where $n \geq 1$, we can define the function $f_{n}: X \rightarrow[0,1]$ by $f_{n}(x)=i / 2^{n}$ when $x \in X_{n}^{i}$ and $i=0,1, \ldots, 2^{n}-1$. Then $f_{n}$ is a continuous step function and $f_{n}(x) \leq$ $f_{n+1}(x) \leq f(x)$ for all $x \in X$ and all $n \geq 1$. Moreover, $f(x)-f_{n}(x) \leq 1 / 2^{n}$ for all $x \in X$. Hence the sequence of step functions $\left\{f_{n}\right\}$ converges uniformly to $f$.

We say that an MV-algebra $A$ is weakly divisible if, given a positive integer $n$, there is an element $v \in A$ such that $n \cdot v=1$, and divisible if, given any $a \in A$ and positive integer $n$, there is an element $v \in A$ such that $n \cdot v=a$. In any case, $A$ has no extremal discrete state. According to (2.3), for $\sigma$-complete MV-algebras, the notions of weak divisibility and divisibility, as well as the property that $A$ admits no discrete (extremal) state, coincide.

THEOREM 5.4. Let $\tau$ be a $\sigma$-complete state-morphism-operator on a weakly divisible $\sigma$-complete $M V$-algebra $A$. If $g$ is the mapping defined in Proposition 3.2, then the operator $\tau_{g}: \hat{A} \rightarrow \hat{A}$, defined by $\tau_{g}(\hat{a})(s)=\hat{a}(g(s))$ for all $a \in A$ and $s \in \partial_{e} \mathcal{S}(A)$, is a $\sigma$-complete state-morphism-operator on $\hat{A}$ such that

$$
\tau_{g}(\hat{a})=(\tau(a))^{\wedge} \quad \forall a \in A .
$$

Proof. By Theorem 2.4, $\hat{A}=M(A)$, where $M(A)$ is defined by (2.3).

Define the operator $\tau_{g}$ on $M(A)$ by $\tau_{g}(f):=f \circ g$ for all $f \in M(A)$. Then $\tau_{g}$ is a state-morphism-operator on $M(A)$, by Proposition 3.5. We will now show that $\tau_{g}=\hat{\tau}$. 
Since $A$ is $\sigma$-complete, $A$ satisfies general comparability. Let $B(A)$ be the set of all Boolean elements of $A$; then $B(A)$ is a Boolean $\sigma$-algebra. In view of Proposition 3.5, the restriction, $\tau_{B}$ of $\tau$ onto $B$ is a state-morphism-operator on $B$. We set $B:=B(A)$. By Theorem 2.3, the state spaces $\partial_{e} \mathcal{S}(A)$ and $\partial_{e} \mathcal{S}(B)$ are homeomorphic basically disconnected compact spaces. Therefore the functions $g$ on $\hat{A}$ and $g_{B}$ on $\hat{B}$ determined by Proposition 3.2 are practically the same, that is, if $\phi_{A}$ is the homeomorphism from Theorem 2.3, then $g_{B} \circ \phi_{A}=\phi_{A} \circ g$. Using Proposition 3.5, we see that $\tau_{g_{B}}=\hat{\tau}_{B}$.

First, let $f$ be a Boolean element in $\hat{A}$. Then $\tilde{f}:=f \circ \phi_{A}^{-1}$ is a Boolean element in $\hat{B}$, and vice versa. Moreover, if $s \in \partial_{e} \mathcal{S}(A)$, then $\tilde{s}=\phi_{A} \circ s=s_{\mid B(A)}$. Consequently,

$$
\hat{\tau} \circ f \circ s=\hat{\tau}_{B} \circ \tilde{f} \circ \tilde{s}=\tilde{f} \circ g_{B} \circ \tilde{s}=f \circ \phi_{A}^{-1} \circ g_{B} \circ \phi_{A} \circ s=f \circ g \circ s,
$$

and so $\hat{\tau}(f)=\tau_{g}(f)$ whenever $f$ is a Boolean element.

Second, since $M(A)$ consists of all continuous functions defined on $\partial_{e} \mathcal{S}(A)$ taking values in the interval $[0,1]$, then if $f \in M(A)$, then $n^{-1} f \in M(A)$ for all positive integers $n$. Suppose that $f$ is a Boolean element from $M(A)$, then $f=n \cdot n^{-1} f$, so $\tau_{g}(f)=\hat{\tau}(f)=n \cdot \hat{\tau}\left(n^{-1} f\right)$. Hence

$$
\hat{\tau}\left(n^{-1} f\right)=n^{-1} \tau_{g}(f)=\tau_{g}\left(n^{-1} f\right)=n^{-1} \hat{\tau}(f) .
$$

Therefore $\tau_{g}((m / n) f)=\hat{\tau}((m / n) f)$ for all integers $m$ between 0 and $n$. Let $t$ be an irrational number in $[0,1]$, and take sequences of rational numbers $r_{n} \nearrow t$ and $s_{n} \searrow t$. Hence

$$
r_{n} \tau_{g}(f)=\tau_{g}\left(r_{n} f\right)=\hat{\tau}\left(r_{n} f\right) \leq \hat{\tau}(t f) \leq \hat{\tau}\left(s_{n} f\right)=\tau_{g}\left(s_{n} f\right)=s_{n} \tau_{g}(f),
$$

so $\tau_{g}(t f)=t \tau_{g}(f)=\hat{\tau}(t f)=t \hat{\tau}(f)$.

Third, let $f \in M(A)$ be a step function, that is, $f=\sum_{i=1}^{n} t_{i} f_{i}$, where each $f_{i}$ is a characteristic function of some clopen set $E_{i}$ and $t_{i} \in[0,1]$ for all $i$. Without loss of generality, we can assume that $E_{1}, \ldots, E_{n}$ are pairwise disjoint. Consequently, $f=t_{1} f_{1}+\cdots+t_{n} f_{n}$, where + is the partial addition in the MV-algebra $M(A)$, which coincides with addition of functions. Hence,

$$
\begin{aligned}
\hat{\tau}(f) & =\hat{\tau}\left(t_{1} f_{1}\right)+\cdots+\hat{\tau}\left(t_{n} f_{n}\right) \\
& =\tau_{g}\left(t_{1} f_{1}\right)+\cdots+\tau_{g}\left(t_{n} f_{n}\right) \\
& =\tau_{g}\left(t_{1} f_{1}+\cdots+t_{n} f_{n}\right)=\tau_{g}(f) .
\end{aligned}
$$

Finally, let $f$ be a continuous function from $M(A)$. By Lemma 5.3, there is a sequence $\left\{f_{n}\right\}$ of continuous step functions from $M(A)$ such that $\left\{f_{n}\right\} \nearrow f$ uniformly. Then $f=\bigvee_{n} f_{n}$. In view of Proposition 3.6, $\hat{\tau}$ is also a $\sigma$-complete state-morphismoperator, so that

$$
\hat{\tau}(f)=\bigvee_{n} \hat{\tau}\left(f_{n}\right)=\bigvee_{n} \tau_{g}(f)=\tau_{g}\left(\bigvee_{n} f_{n}\right)=\tau_{g}(f),
$$

by the argument of the previous paragraph. 


\section{Acknowledgements}

The authors are indebted to the referees for their careful reading and suggestions.

\section{References}

[1] G. Barbieri and H. Weber, 'Measures on clans and on MV-algebras', in: Handbook of Measure Theory, Vol. II (ed. E. Pap) (Elsevier Science, Amsterdam, 2002), pp. 911-945.

[2] D. Buhagiar, E. Chetcuti and A. Dvurečenskij, 'Loomis-Sikorski representation of monotone $\sigma$ complete effect algebras', Fuzzy Sets and Systems 157 (2006), 683-690.

[3] C. C. Chang, 'Algebraic analysis of many-valued logics', Trans. Amer. Math. Soc. 88 (1958), $467-490$.

[4] R. Cignoli, I. M. L. D'Ottaviano and D. Mundici, Algebraic Foundations of Many-valued Reasoning (Kluwer Academic Publishers, Dordrecht, 1998).

[5] A. Di Nola and A. Dvurečenskij, 'State-morphism MV-algebras', Ann. Pure Appl. Logic 161 (2009), 161-173.

[6] A. Di Nola and A. Dvurečenskij, 'On some classes of state-morphism MV-algebras', Math. Slovaca 59 (2009), 517-534.

[7] A. Di Nola, A. Dvurečenskij and A. Lettieri, 'On varieties of MV-algebras with internal states', Internat. J. Approx. Reason. 51 (2010), 680-694.

[8] A. Di Nola, A. Dvurečenskij and A. Lettieri, 'Erratum state-morphism MV-algebras', Ann. Pure Appl. Logic 161 (2010), 1605-1607, Ann. Pure Appl. Logic 161 (2009), 161-173.

[9] A. Dvurečenskij, 'Loomis-Sikorski theorem for $\sigma$-complete MV-algebras and $\ell$-groups', J. Aust. Math. Soc. Ser. A 68 (2000), 261-277.

[10] A. Dvurečenskij, 'States on pseudo-effect algebras with general comparability', Kybernetika (Prague) 40 (2004), 397-420.

[11] A. Dvurečenskij and G. Kalmbach, 'States on pseudo MV-algebras and the hull-kernel topology', Atti Semin. Mat. Fis. Univ. Modena Reggio Emilia 50 (2002), 131-146.

[12] A. Dvurečenskij and S. Pulmannová, New Trends in Quantum Structures (Kluwer Academic Publishers, Dordrecht, 2000).

[13] A. Dvurečenskij and J. Rachůnek, 'On Riečan and Bosbach states for bounded non-commutative $R \ell$-monoids', Math. Slovaca 56 (2006), 487-500.

[14] T. Flaminio and F. Montagna, 'An algebraic approach to states on MV-algebras', in: Fuzzy Logic 2, Proc. 5th EUSFLAT Conference, Ostrava, 11-14 September 2007 (ed. V. Novák) (Universitas Ostraviensis, Ostrava, 2007), Vol. II, pp. 201-206.

[15] T. Flaminio and F. Montagna, 'MV-algebras with internal states and probabilistic fuzzy logic', Internat. J. Approx. Reason. 50 (2009), 138-152.

[16] G. Georgescu, 'Bosbach states on fuzzy structures', Soft Computing 8 (2004), 217-230.

[17] K. R. Goodearl, Partially Ordered Abelian Groups with Interpolation, Mathematical Surveys and Monographs, 20 (American Mathematical Society, Providence, RI, 1986).

[18] J. Jakubík, 'On complete MV-algebras', Czechoslovak Math. J. 45(120) (1995), 473-480.

[19] T. Kroupa, 'Every state on semisimple MV-algebra is integral', Fuzzy Sets and Systems 157 (2006), $2771-2782$.

[20] J. Kühr and D. Mundici, 'De Finetti theorem and Borel states in [0, 1]-valued algebraic logic', Internat. J. Approx. Reason. 46 (2007), 605-616.

[21] K. Kuratowski, Topology I (Mir, Moskva, 1966) (in Russian).

[22] L. H. Loomis, 'On the representation of $\sigma$-complete Boolean algebras', Bull. Amer. Math. Soc. 53 (1947), 757-760.

[23] D. Mundici, 'Interpretation of AF $C^{*}$-algebras in Łukasiewicz sentential calculus', J. Funct. Anal. 65 (1986), 15-63.

[24] D. Mundici, 'Averaging the truth-value in Łukasiewicz logic', Studia Logica 55 (1995), 113-127.

[25] D. Mundici, 'Tensor products and the Loomis-Sikorski theorem for MV-algebras', Adv. Appl. Math. 22 (1999), 227-248. 
[26] G. Panti, 'Invariant measures in free MV-algebras', Comm. Algebra 36 (2008), 2849-2861.

[27] B. Riečan, 'On the probability on BL-algebras', Acta Math. Nitra 4 (2000), 3-13.

[28] B. Riečan and D. Mundici, 'Probability on MV-algebras', in: Handbook of Measure Theory, Vol. II (ed. E. Pap) (Elsevier Science, Amsterdam, 2002), pp. 869-909.

[29] R. Sikorski, 'On the representation of Boolean algebras as fields of sets', Fund. Math. 35 (1948), $247-256$.

[30] R. Sikorski, Boolean Algebras, 2nd edn, Ergebnisse der Mathematik und ihrer Grenzgebiete, Folge 2, 25 (Springer, Berlin-Göttingen-Heidelberg, 1964).

A. DI NOLA, Dipartimento di Matematica e Informatica, Università di Salerno, Via Ponte don Melillo, I-84084 Fisciano, Salerno, Italy

e-mail: adinola@unisa.it

A. DVUREČENSKIJ, Mathematical Institute, Slovak Academy of Sciences, Štefánikova 49, SK-814 73 Bratislava, Slovakia

e-mail: dvurecen@mat.savba.sk

A. LETTIERI, Dipartimento di Costruzioni e Metodi Matematici in Architettura, Università di Napoli Federico II, via Monteoliveto 3, I-80134 Napoli, Italy e-mail: lettieri@unina.it 